are shown in Table 1. All arteries except the carotid arteries had a significantly higher IMT in the LV-GCA and mixed-GCA groups when compared with both C-GCA and non-GCA groups. There were no differences in IMT between mixed-GCA and LV-GCA. There were also no differences in any explored artery between PMR and non-GCA. There were statistically significant differences in the IMT of the bilateral axillary and subclavian arteries between the PMR group and all the GCA subtypes, being greater in the latter. IMT tended to be higher in the C-GCA group when compared to non-GCA, reaching statistical significance in the left arteries (axillary, subclavian, and distal carotid). Although there was also a tendency for IMT to be higher in mixedGCA patients than in LV-GCA patients, the differences did not reach statistical significance.

Conclusion: Large vessel ultrasound does not differ between healthy patients and those with PMR without confirmed GCA. Our data suggest that mixed-GCA subtype is not an intermediate form between the cranial and LV-GCA suptypes but could have a higher inflammatory burden.

Disclosure of Interests: Elisa Fernández-Fernández: None declared, Iñigo González-Mazón: None declared, Irene Monjo Speakers bureau: Roche, Novartis, UCB, Gedeon Richter, Consultant of: Roche, José María Mostaza: None declared, Carlos Lahoz: None declared, Eugenio de Miguel Speakers bureau: AbbVie, Novartis, Pfizer, MSD, BMS, UCB, Roche, Grunental, Janssen, Sanofi, Paid instructor for: Janssen, Novartis, Roche, Consultant of: AbbVie, Novartis, Pfizer, Galapagos, Grant/research support from: Abbvie, Novartis, Pfizer

DOI: 10.1136/annrheumdis-2021-eular.4134

\section{AB0383 OCULAR MANIFESTATIONS DURING GIANT CELL ARTERITIS}

A. Mzabi ${ }^{1}$, N. Adaily ${ }^{1}$, L. Ines ${ }^{1}$, I. Ben Hassine ${ }^{1}$, J. Anoun ${ }^{1}$, M. Karmani ${ }^{1}$, F. Ben Fredj ${ }^{1}$, C. Laouani ${ }^{1}{ }^{1}$ University Hospital Center of Sahloul, Internal Medicine, Sousse, Tunisia

Background: Horton's disease (HD) or giant cell arteritis (GCA) is a segmental and plurifocal giant cell inflammatory panarteritis, predominant in large and medium sized vessels. Ocular damage during giant cell arteritis is frequent and is considered the most serious damage, possibly causing blindness in $15 \%$ of cases in recent series (1).

Objectives: Describe the different ophthalmological manifestations during GCA.

Methods: This is a retrospective monocentric descriptive study of 19 files of subjects diagnosed with $\mathrm{HD}$, hospitalized in the internal medicine department of de Sousse between January 2000 and December 2020.

Results: The average age of patients at diagnosis was 70 years, with extremes of 53 and 92 years. There were 14 females $(71.4 \%)$ and 5 males $(28.6 \%)$. Ophthalmologic manifestations were present in 13 patients $(71 \%), 6$ of whom were female.

Decreased visual acuity was reported in 8 cases (58.33\%) with biolateral blindness in one case, visual blur in 9 cases $(75 \%)$, eye pain in two cases.

Ophthalmological examination was normal in 8 cases $(42 \%)$. A decrease in visual acuity was objective in 4 cases $(21 \%)$. One case of nodular episcleritis was noted. The fundus showed anterior ischemic optic neuropathy in 2 cases $(10.5 \%)$ and left optic atrophy in another case. A visual field was performed in 2 cases showing associated retrobulbar optic neuritis. Therapeutically, Methylprednisolone boli were prescribed in patients with anterior ischemic optic neuropathy, followed by oral corticosteroid therapy at a dose of $1 \mathrm{mg} / \mathrm{kg} / \mathrm{day}$. A dose of $0.7 \mathrm{mg} / \mathrm{kg} /$ day of prednisone was prescribed in the other cases. Blindness was irreversible.

Conclusion: The most common eye diseases in HD are acute anterior ischemic optic neuritis, retinal artery occlusion and posterior ischemic optic neuropathy. Episcleritis is a rare ophthalmologic manifestation of this vasculitis (2). More exceptionally, Horton's disease may cause NORB by affecting the vessels that supply the optic nerve $(<5 \%$ of cases $)(3,4)$. In our series of studies, acute anterior ischemic optic neuritis was less observed than in the literature. This is probably due to the reduced number of patients.

\section{REFERENCES:}

[1] M.Khedher. I.Rachdi. Z.Aydi. F.Daoud. B.Ben Dhaou. F.Boussema. Eye damage during Horton's disease: about 42 cases. La Revue de Médecine Interne 2017; 38:115

[2] Rim Klii. Wafa Chebbi. Nodular episcleritis: an unusual inaugural manifestation of Horton's disease. Pan Afr Med J 2015; 21: 20.

[3] M.Essouril.RachdiF.DaoudZ.AydiH.ZoubeidiB.Ben Dhaou et al. Retrobulbar Optic Neuropathy in Internal Medicine. La Revue de Médecine Interne 2018; 39: 132.

[4] M A González-Gay, C García-Porrúa, J Llorca, A H Hajeer, F Brañas, A Dababneh et al. Visual manifestations of giant cell arteritis. Trends and clinical spectrum in 161 patients. Medicine (Baltimore) 2000;79(5): 283-92.

Disclosure of Interests: None declared

DOI: 10.1136/annrheumdis-2021-eular.4143

\section{\begin{tabular}{|l|l}
\hline AB0384 IMPACT OF TEMPORAL ARTERY ULTRASOUND \\
\hline
\end{tabular} ON SURVIVAL OF PATIENTS WITH GIANT CELL ARTERITIS}

P. Estrada ${ }^{1}$, D. Reina ${ }^{1}$, V. Navarro ${ }^{1}$, O. Camacho ${ }^{1}$, D. Cerda ${ }^{1}$, D. Roig Vilaseca ${ }^{1}$, S. Heredia ${ }^{1}$, H. Corominas ${ }^{2}{ }^{1}$ Hospital de Sant Joan Despí Mois ès Broggi, Rheumatology, Sant Joan Despí, Spain; ${ }^{2}$ Hospital de la Santa Creu i Sant Pau, Rheumatology, Barcelona, Spain

Background: Giant cell arteritis (GCA) is the most common primary systemic vasculitis in adults over 50 years of age. Its incidence increases with age, with a peak between $70-80$ years and predominates in women, 3:1. It is a medical emergency that, if not diagnosed, can lead to irreversible complications. The delay in time from diagnosis to start of treatment is crucial to avoid possible serious outcomes on short, medium and long term. Survival in GCA is estimated between $60-90 \%$ at 5 years and $48-81 \%$ at 10 years. Efforts have been made to implement rapid diagnostic circuits to assess patients and initiate treatment without delay with good results both in reducing permanent vision loss and in reducing the costs of these patients due to emergency visits and admissions. The morbidity and mortality of this disease is high, but the use of efficient diagnostic strategies, such as ultrasound of superficial temporal arteries, has proven to be a useful, practical, cost-effective and, above all, quick tool to make the diagnostic approach.

Objectives: Analyze the impact of early temporal artery ultrasound on survival for patients with GCA.

Methods: Survival study of 48 patients with GCA, in two different "stages" in terms of diagnostic approach: Group A $(n=27)$, patients diagnosed between 2002 - 2011 using only ACR 1990 criteria and Group $E(n=21)$ diagnosed between 2010-2015 using ACR criteria and TAUS. TAUS was performed by Rheumatologists with extensive experience in ultrasound and within a period of no more than 7 days for these patients. The definitive diagnosis of GCA was based on the clinical criteria of the Rheumatologist within the clinical and analytical context and with the specific complementary examinations for each case (Ultrasound, PET-CT, biopsy). Demographic data, comorbidities, signs and symptoms at debut, analytical data, complementary examinations, treatment and evolution were obtained retrospectively through the electronic medical record of the patient, based on the database of our GCA cohort. A survival analysis was performed considering death as the main outcome. The statistic used was the Kaplan-Meier test. In addition, other complications related to treatment or pathology are collected.

Results: The mean age at diagnosis of our patients was $79+-6$ years, with a female: male ratio of $3: 1$. The follow-up was between 2 and 16 years with a mean of $5.8+-3$ years, until the last visit collected or until the outcome of death. Group A had a survival at 5 and 10 years of $53.4 \%$ and $36.7 \%$ respectively, while group E of $79.5 \%$ at both cut-off points. (Figure 1 ).

There is a significant difference between the survival of both groups, $p$ $<0.01$, this being better in the group in which TAUS was implemented for rapid diagnosis (group $\mathrm{E}$ ). The main causes of death were cardiovascular 
events, $30 \%$, predominantly in group $E(75 \%)$, and infection, $30 \%$, predominantly in group $\mathrm{A}$. The median from diagnosis to death was 3 years (range $1-13)$.

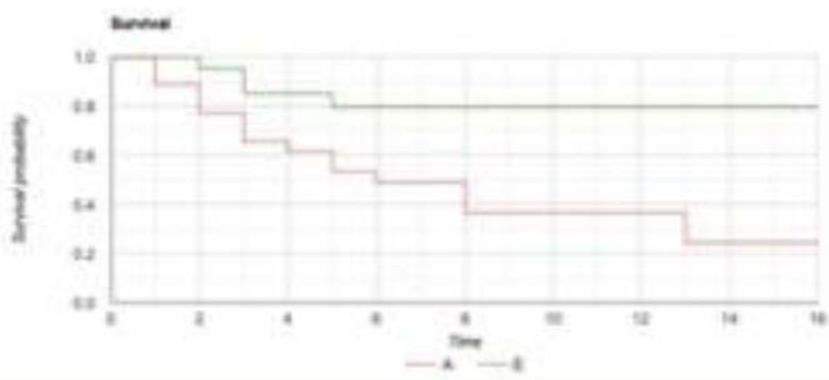

Figure 1. Group A (red line) according to ACG 1990 criteria and Group B (green line) according to ACG criteria and implementing TAUS for rapid diagnosis

Conclusion: The implementation of temporal artery ultrasound (TAUS) is associated with a significant improvement in the survival rate of patients with GCA and a reduction in treatment-related complications in patients who were diagnosed with ultrasound in less than 7 days compared to those diagnosed by the conventional healthcare attention routes.

REFERENCES:

[1] Gonzalez-Gay MA, et al. Giant cell arteritis: epidemiology, diagnosis, and management. DOI: 10.1007/s11926-010-0135-9

[2] Patil $\mathrm{P}$, et al. Fast track pathway reduces sight loss in giant cell arteritis: results of a longitudinal observational cohort study. PMID: 26016758

[3] Breuer GS, et al. Survival of patients with giant cell arteritis: a controversial issue. PMID: 31969222

[4] Diamantopoulos AP, et al. The fast-track ultrasound clinic for early diagnosis of giant cell arteritis significantly reduces permanent visual impairment: towards a more effective strategy to improve clinical outcome in giant cell arteritis? 10.1093/rheumatology/kev289

Disclosure of Interests: None declared

DOI: 10.1136/annrheumdis-2021-eular.4158

\section{Vasculitis - small vessel vasculitis}

\section{AB0385 THE EFFECTIVENESS OF THE ANTI-TUMOR NECROSIS FACTOR THERAPY INFLIXIMAB IN NEURO- BEHCET'S DISEASE: A SYSTEMATIC REVIEW AND META-ANALYSIS}

R. Hamdy Abdellatif Mohammed ${ }^{1}$, Y. Woldeamanuel ${ }^{2}{ }^{1}$ Kasr Alainy School of Medicine- Cairo University., Rheumatology and Rehabititation, Cairo, Egypt; ${ }^{2}$ Stanford University, Stanford Headache Center, Department of Neurology- Center for Innovation in Global Health- Stanford University School of Medicine: Stanford, CA, US., Stanford-CA, United States of America

Background: The use and benefit of biologic targeted therapy in the treatment of refractory manifestations of behcet's syndrome remain unclearly defined due to the relative paucity of published research. Neuro-behcet's disease represents a challenging clinical presentation. The lack of identified effective strategies in the management of refractory neuro-behcet's adds to the disease burden and contributes to lack of tight control. [1-3]

Objectives: The aim of this systematic review and meta-analysis is to examine the effect of anti-TNF therapy on refractory NBD by performing a systematic review and meta-analysis of the previously published studies assessing the effectiveness of anti-TNF therapy in patients with BD having refractory or recurrent neurological manifestations.

Methods: Authors used the PICO Model $(\mathrm{P}=$ population, $\mathrm{I}=$ intervention, $\mathrm{C}=$ comparator, $\mathrm{O}=$ outcome) used for designing the research question. The PRISMA statement was used for developing the study protocol search methodology. Literature searches were done for articles in published in English language only from January 2000 till January 2020 (considering the first till the last available publication that addresses the research question and satisfied eligibility criteria).
The study was registered on the Prospero PROSPERO website for systematic reviews and meta-analysis. Random-effects meta-analysis was performed. Interstudy heterogeneity was explored using 12 statistics. Cumulative meta-analysis was conducted to assess temporal trend for accumulating evidence from accruing published studies. Meta-regression was used to analyze possible confounders. Authors of the current systematic review and meta-analysis worked on a simple score "Hamdy and Woldeamanuel simple response score, 2020", the aim behind the score was to incorporate standard evaluation parameters for assessment of responses driven by the measures used in each study. The authors used numerical values to grade the pattern of response in each parameter considered attempting to standardize the quantification of the responses and minimize tendency towards overestimation. The score included clinical and radiographic points rated on a scale of 5

Results: Twenty-one studies involving 64 patients were included, 52 male patient and 12 females at a ratio of 4.3:1, with a mean age of 38.21 years, mean disease duration of 84.765 months. Effect size analysis showed that 59 out of 64 patients i.e. $93.7 \%$ of the treated patients with neuro-behcet's disease in the analysis were responders to infliximab therapy (Cl 88\% - 99.3\%) There was no significant inter-study heterogeneity $(I 2=0 \%, p=0.744)$. Cumulative analysis showed accumulating evidence favoring increasing effectiveness over the last 20 years. There was no statistically significant confounding of infliximab effect size by age $(p=0.89)$, sex ratio $(p=0.29)$, and disease duration $(p=0.67)$

Conclusion: In this systematic review and meta-analysis Infliximab showed a strong therapeutic effectiveness in the treatment of refractory neuro-behcet's disease. There is an increasing trend of effectiveness observed in the last two decades which may be due to improved neuro-behcet's diagnostic accuracy.

\section{REFERENCES:}

[1] Uygunoğlu U, Siva A. Behçet's Syndrome and Nervous System Involvement. Curr Neurol Neurosci Rep. 2018;18(7):35. Published 2018 May 23. doi:10.1007/s11910-018-0843-5.

[2] Ohno S, Ohguchi M, Hirose S, Matsuda H, Wakisaka A, Aizawa M. Close Association of HLA-Bw51 With Behçet's Disease. Arch Ophthalmol. 1982; 100(9):1455-1458. doi:10.1001/archopht.1982.01030040 433013.

[3] Kalra, S., Silman, A., Akman-Demir, G., et al. Diagnosis and management of Neuro-Behçet's disease: international consensus recommendations. Journal of neurology, 2014; 261(9): 1662-1676. https://doi.org/10.1007/ s00415-013-7209-3.

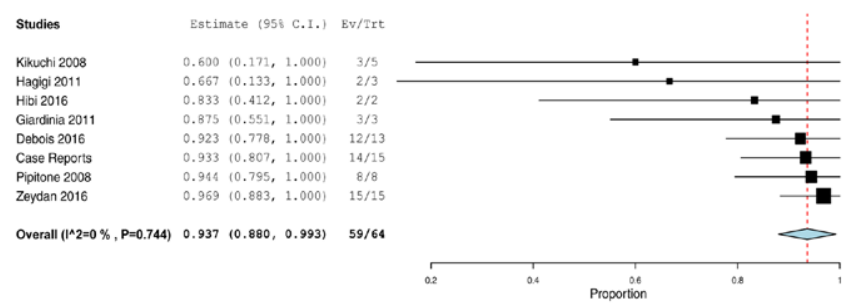

Figure 1 a. Forest plot summarizing the meta-analysis results on the effectiveness of infliximab to treat Neurobehcet disease. Overall, $94 \%$ of patients responded to infliximab.

Disclosure of Interests: None declared

DOI: 10.1136/annrheumdis-2021-eular.52

\section{AB0386 CORONAVIRUS INFECTION AND VASCULITIS: IDENTIFYING ASSOCIATIONS MINING THE BIOMEDICAL LITERATURE}

A. Rodriguez-Pla ${ }^{1}$, R. Cartin-Ceba ${ }^{2} .{ }^{1}$ Mayo Clinic Scottsdale Campus, Rheumatology, Scottsdale, United States of America; ${ }^{2}$ Mayo Clinic Hospital, Pulmonary, Phoenix, United States of America

Background: Based on recent publications suggesting an association between COVID-19 and vascular

Inflammation.

Objectives: Our aim was to explore new associations between coronavirus infections and vasculitis utilizing semantic mining of PubMed results.

Methods: The following literature search string: "(vasculitis OR vascular inflammation OR vascular damage) AND (coronavirus OR SARS virus OR 